\title{
Tamil Version of the Fear of COVID-19 Scale
}

\author{
Jaishankar Bharatharaj ${ }^{1}$ (D) Mohsen Alyami ${ }^{2}$ (D) Marcus A. Henning ${ }^{3}$ (D) \\ Hussain Alyami $^{4}$ (D) Christian U. Krägeloh ${ }^{5,6}$ (D)
}

Accepted: 8 March 2021/ Published online: 16 June 2021

(C) The Author(s), under exclusive licence to Springer Science+Business Media, LLC, part of Springer Nature 2021

The novel coronavirus outbreak in December 2019 (COVID-19), caused by severe acute respiratory syndrome coronavirus 2 (SARS-CoV-2), had spread to 213 countries and territories since December 2019 until the time of writing this paper (Worldometer, 2020). As of 28 June 2020, there are 10,012,299 confirmed cases and a reported death toll of 499,185 worldwide. In India, the number of COVID-19 cases is growing exponentially with 505,953 cases and 15,685 deaths (Johns Hopkins University \& Medicine, 2020). The state of Maharashtra, Delhi, and the state of Tamil Nadu have the highest number of confirmed COVID-19 cases, respectively (Johns Hopkins University \& Medicine, 2020).

The impact of the pandemic itself and the measures taken to contain the spread of COVID19 (e.g., quarantine) and their subsequent social and economic consequences on mental health and well-being is likely to be significant (Galea et al., 2020; Rajkumar, 2020). A recent review found that anxiety and depression symptoms, stress, and disturbed sleep were common manifestations of the COVID-19 pandemic (Rajkumar, 2020).

Research has also shown heightened levels of fear of COVID-19 among various populations (Knipe et al., 2020), including healthcare professionals ( $\mathrm{Lu}$ et al., 2020), similar to previous viral epidemics (Bukhari et al., 2016; Reynolds et al., 2008). Fear of COVID-19 could worsen mental health and well-being (e.g., among university students, see Gritsenko et al., 2020), or it could motivate individuals to comply with the recommended health measures (Harper et al., 2020; Winter et al., 2020).

Ahorsu et al. (2020) recently developed the Fear of COVID-19 Scale (FCV-19S) which measures the severity of fear of COVID-19. The FCV-19S is a unidimensional seven-item

Christian U. Krägeloh

chris.krageloh@aut.ac.nz

1 PAIR Lab, Bharath Institute for Higher Education and Research, Chennai, India

2 Department of Psychological Medicine, University of Auckland, Auckland, New Zealand

3 Centre of Medical and Health Sciences Education, University of Auckland, Auckland, New Zealand

4 College of Medicine, Taif University, Taif, Saudi Arabia

5 PAIR Lab, Auckland University of Technology, Auckland, New Zealand

6 Department of Psychology and Neuroscience, Auckland University of Technology, Auckland, New Zealand 
scale with robust psychometric properties (Ahorsu et al., 2020). Using the English version of the FCV-19S, a recent study (Doshi et al., 2020) examined the level of fear of COVID-19 among 1499 Indian residents and found that more than half of the sample (54.8\%) reported low levels of fear. In this study, participants who were female, married, had lower educational status, and were working in healthcare had significantly higher odds of reporting higher levels of fear of COVID-19 (Doshi et al., 2020). To accurately assess fear of COVID-19, the reliability and validity of the FCV-19S need to be first evaluated in the targeted population, particularly in their preferred language. In the present study, the language that we focused on is Tamil, which is spoken by over 70 million people worldwide. Most native speakers reside in Tamil Nadu, an Indian southern state with a population of over 72 million people according to the 2011 Census (National Informatics Centre, 2020). Tamil is also an official language of Sri Lanka and Singapore, where it is spoken by $25 \%$ (Encyclopedia.com, 2021) and 3-4\% (Department of Statistics Singapore, 2021) of the population, respectively, but it is also widely spoken in Malaysia, Mauritius, Fiji, and South Africa (Britannica.com, 2021). The present study developed a Tamil version of the FCV-19S and assessed its psychometric properties using Rasch analysis. This scale will thus enable studies exploring the psychosocial impact of COVID-19 in Tamil-speaking populations.

\section{Methods}

\section{Participants}

Participants were Tamil-speaking adult Indian citizens residing in the state of Tamil Nadu since the COVID-19 pandemic was declared by the World Health Organization. The respondents were able to read and write in the Tamil language and were aged between 18 and 67 years. A total of 95 responses was received. Due to a programming error, 25 participants had no data available for age, 33 no data for education, and 4 no data for gender. With the available data on remaining participants, the mean age was 31.07 years $(\mathrm{SD}=10.25)$ with $46(51 \%)$ male and $45(49 \%)$ female participants. Ten respondents indicated that they had completed their schooling, 40 had graduated from a higher-education institute, and 11 participants held a postgraduate qualification.

\section{Procedure}

Participants were recruited using a snowball sampling method to complete an anonymous online survey. The research was advertised via social media platforms (Facebook and LinkedIn), personal and professional networks through email with detailed aims and objectives of the study. The Google Forms' link was included in the advertisement, and instructions on completing the survey were provided. Participants were also requested to share the survey link with their personal and professional contacts. Answering all questionnaire items was mandatory to submit. The questionnaire also contained a brief section on demographic information (gender, age, and education). Data reported in this study were collected from $31^{\text {st }}$ of May to $21^{\text {st }}$ of June 2020. Participation in the study was voluntary, and no reward or compensation was provided. The study was approved by the first author's institutional ethics committee. 


\section{Adaptation of FCV-19S into Tamil}

Following established protocols for cross-cultural adaptation (Beaton et al., 2000), the original FCV-19S was translated from English into Tamil using forward-backward translation. First, the questionnaire was translated into Tamil by an independent translator fluent in both English and Tamil and with a post-graduate qualification. Second, the first author, who is fluent in English and Tamil, reviewed the provisional Tamil translation and discussed any concerns with the independent translator. Third, after approval of the Tamil translation, the preliminary Tamil version was back-translated into English by an independent native speaker of Tamil, with a post-graduate qualification in English who at that time was unfamiliar with the original scale. At the final stage, any discrepancies were discussed with the team of translators. These were very minor variations that did not influence the meaning of the item. The final Tamil version of the FCV-19S can be found in the Appendix.

\section{Measures}

\section{Fear of COVID-19}

The seven-item FCV-19S (Ahorsu et al., 2020) measures respondents' fear levels of COVID19. All items are positively worded, implying that higher scores indicate greater levels of fear. Respondents rate items on a 5-point Likert scale, ranging from 1 (strongly disagree) to 5 (strongly agree). Most validation studies have proposed a unidimensional solution, which allows the calculation of a total score (range 7 to 35) by summing all seven items. The scale has shown robust psychometric properties in its various language versions (Ahorsu et al., 2020; Alyami et al., 2020; Bitan et al., 2020; Doshi et al., 2020; Huarcaya-Victoria et al., 2020; Mahmood et al., 2020; Masuyama et al., 2020; Pang et al., 2020; Perz et al., 2020; Reznik et al., 2020; Sakib et al., 2020; Satici et al., 2020; Soraci et al., 2020; Tsipropoulou et al., 2020; Zolotov et al., 2020) including high internal consistency (Cronbach's alpha ranging from .81 to .91).

\section{Statistical Analysis}

Descriptive statistics were conducted using IBM SPSS Statistics v 26 software. Item-level analyses included means, standard deviations, skewness, and kurtosis. Internal consistency was assessed by Cronbach's alpha $(\alpha)$ coefficients and the impact on alpha if each item was deleted. Inter-item correlations and corrected item-total correlations further explored associations between items, where coefficients between .30 and .70 suggest medium to strong associations (Ferketich, 1991).

Detailed psychometric properties of the Tamil version of the FCV-19S were investigated using partial credit Rasch analysis with the software RUMM2030 (Andrich et al., 2009). Given the small number of items in the scale, the present sample size of 95 was considered sufficient (Willse, 2017), and the analyses provided 95\% confidence that the item locations would be within \pm 0.50 logits (Linacre, 1994). A likelihood ratio test confirmed the suitability of the present dataset for the partial credit model $\left(\chi^{2}(17)=41.48, p<.001\right)$. Rasch analysis commenced with a baseline model of all seven items. Satisfactory fit was indicated by a nonsignificant ( $p>.05$, Bonferroni adjusted) chi-square for item-trait interaction (Krägeloh et al., 2019). Solutions were inspected for significant item misfit and differential item 
functioning by demographic factors. Given a previous proposal for a two-factor solution of the FCV-19S (Bitan et al., 2020), residual correlations were monitored for patterns that could indicate the presence of multidimensionality. After a nonsignificant Rasch fit had been achieved, unidimensionality was tested using Smith's (2002) test. As a measure of internal consistency reliability, Rasch analysis provides the Person Separation Index (PSI). The value is interpreted in a similar way as Cronbach's $\alpha$, where values of .70 or higher indicate acceptable reliability (DeVellis, 2016). While a minimum PSI of .70 is required for group comparisons of scale scores, values of .85 and above indicate that the scale is suitable for within-participant analyses such as comparisons of change scores (Tennant \& Conaghan, 2007).

\section{Results}

Table 1 shows descriptive statistics for the Tamil version of the FCV-19S. Skewness values ranged from -0.79 to 0.59 . Kurtosis was mostly negative, with the exception of item 7 (1.50), which also had a value that is at the cusp of the recommended acceptable range of -1.50 to 1.50 (Byrne \& Campbell, 1999). Overall Cronbach's alpha was 0.93, and Cronbach's alpha values when deleting each item decreased to either .91 or .92 .

A comparative overview of means, skewness, and kurtosis with those found in 13 other samples around the world is provided in Table 2. Overall, the data from the present Tamil version demonstrate less skewness and kurtosis than most other comparison datasets. High skewness and kurtosis such as found in the two New Zealand samples (Winter et al., 2020) and one of the studies reporting data from a Hebrew version (Bitan et al., 2020) were related to low means and possible floor effects for items 3,6, and 7. The pattern of item means of the Tamil version appeared to be most similar to that of the Bangla version (Sakib et al., 2020) and also similar to that found for the English-language version used in a sample in India (Doshi et al., 2020).

Table 3 shows inter-item correlation coefficients as well as corrected item-total correlation coefficients for each item. All items were moderately to highly correlated with each other, and item-total correlation ranged from .68 to .84 . Item 7 was highly correlated $(r=.79)$ with items 3 and 6 . While this may indicate a potential item cluster, the pattern was not very clear from this analysis, as item 7 was also highly correlated $(r=.72)$ with item 4 . Additionally, correlations between other items with items 3,6, and 7 were not generally lower than correlations among items $1,2,4$, and 5 .

Table 1 Descriptive details for the Tamil FCV-19S (overall Cronbach alpha score $=0.93$ )

\begin{tabular}{lrrrr}
\hline Item & Mean (SD) & Skewness & Kurtosis & Cronbach alpha when item deleted \\
\hline 1 & $3.45(1.24)$ & -0.79 & -0.46 & .92 \\
2 & $3.35(1.27)$ & -0.65 & -0.71 & .92 \\
3 & $2.37(1.35)$ & 0.56 & -1.05 & .91 \\
4 & $2.75(1.47)$ & 0.16 & -1.42 & .91 \\
5 & $3.16(1.45)$ & -0.32 & -1.36 & .91 \\
6 & $2.44(1.47)$ & 0.59 & -1.15 & .91 \\
7 & $2.66(1.53)$ & 0.28 & 1.50 & .91 \\
Total score & $20.18(8.13)$ & -0.06 & -1.26 & - \\
\hline
\end{tabular}




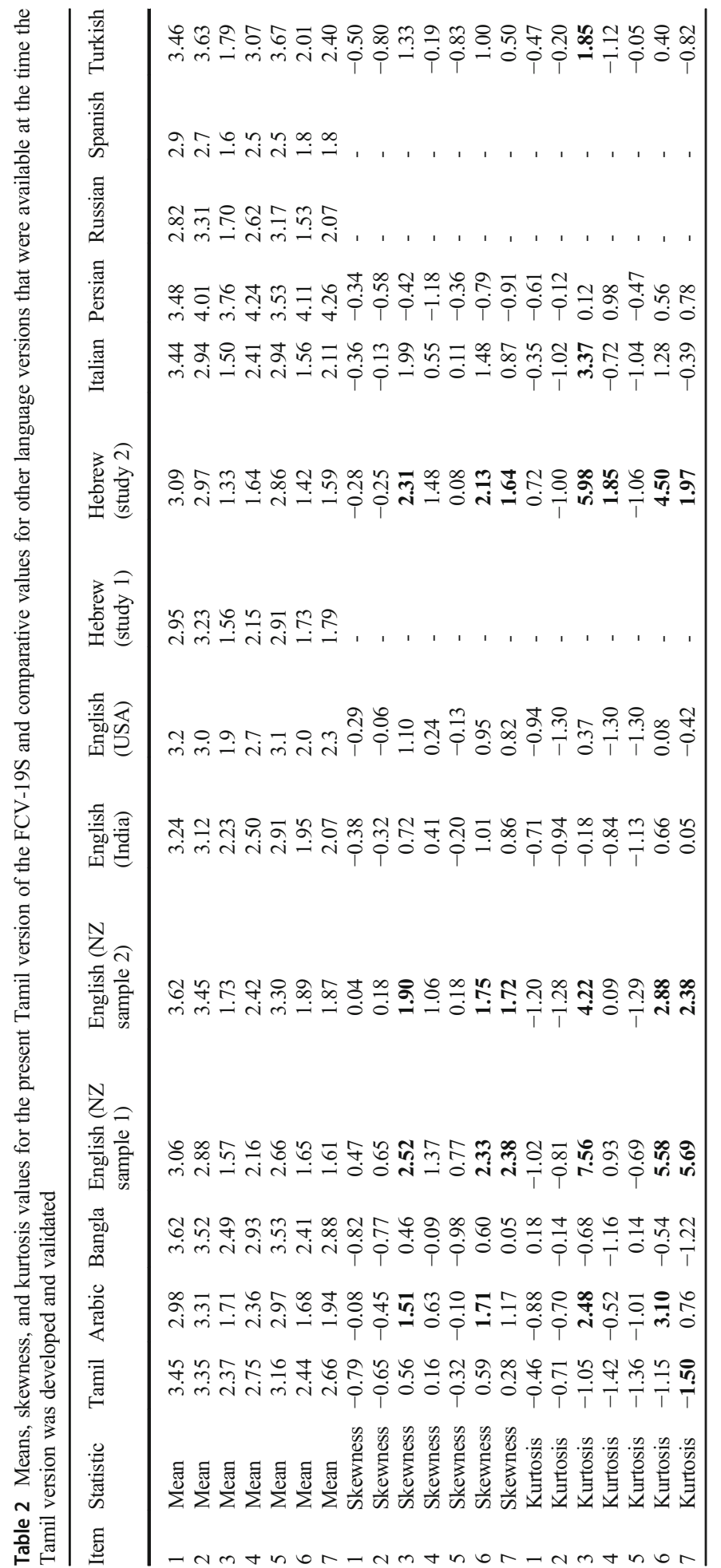

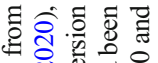

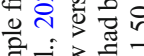

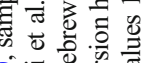

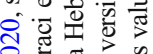

ते

䟠

可记

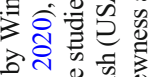

空

당

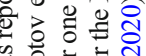

\& 흥 훙

군

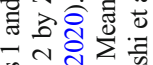

$\checkmark$ N $\sum^{2}$

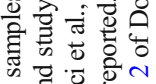

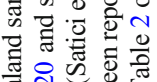

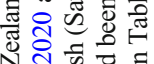

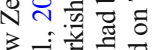

就普政

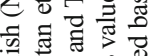

क力 की

宅命领

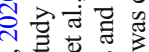

ब

o 응 है

戛

政

뜽

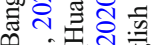

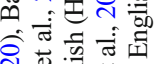

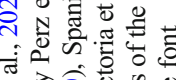

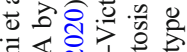

青出

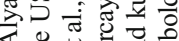

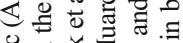

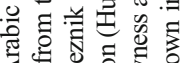

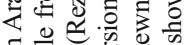

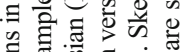

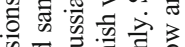

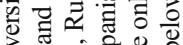

क्षेत्र की

क्ते ते

ส ส

$\Rightarrow$ 政

응

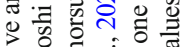

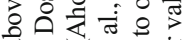


Table 3 Inter-item Pearson's correlation matrix and corrected item-total correlations

\begin{tabular}{|c|c|c|c|c|c|c|c|c|}
\hline Item & Item 1 & Item 2 & Item 3 & Item 4 & Item 5 & Item 6 & Item 7 & Corrected item-total correlations \\
\hline 1 & - & & & & & & & .70 \\
\hline 2 & $.67 *$ & - & & & & & & .68 \\
\hline 3 & $.54 *$ & $.55 *$ & - & & & & & .78 \\
\hline 4 & $.58^{*}$ & $.53 *$ & $.65^{*}$ & - & & & & .78 \\
\hline 5 & $.66^{*}$ & $.62 *$ & $.56^{*}$ & $.73 *$ & - & & & .78 \\
\hline 6 & $.54 *$ & $.56 *$ & $.77 *$ & $.66^{*}$ & $.61 *$ & - & & .79 \\
\hline 7 & $.58 *$ & $.57 *$ & $.79 *$ & $.72 *$ & $.71 *$ & $.79 *$ & - & .84 \\
\hline
\end{tabular}

*Statistically significant at $p<.01$ (2-tailed)

The baseline model of the Rasch analysis already provided a satisfactory fit $\left(\chi^{2}(14)=16.85\right.$, $p>.05$ ), with no evidence of differential item functioning by demographic variables. None of the items was misfitting, and all were well within the acceptable range of -2.50 to 2.50 . Due to a previous report of a two-factor structure of the scale (Bitan et al., 2020), the results were inspected for any indications of multidimensionality in the present dataset. The residual correlation coefficients for items 3,6, and 7 exceeded the mean value of all such residual correlations by at least 0.20 , indicating some local response dependency. However, since the overall fit was satisfactory, this local dependency was not sufficient to warrant postulating a second factor. Smith's (2002) test indicated that the seven-item solution was unidimensional. The internal consistency of this unidimensional solution was excellent. PSI was .88, indicating that the scale is suitable for between-group as well as within-group comparative analyses. The only issue highlighted was in the person-item distribution. As shown in Fig. 1, items failed to provide enough coverage for the bottom $7 \%$ of the sample. This floor effect indicates that the scale is unsuitable to provide detailed differentiation between participants at the very low end of the fear of COVID-19 spectrum.

\section{Discussion}

The present study translated the FCV-19S into Tamil and tested its psychometric properties using Rasch analysis. The data fit the unidimensional Rasch model very

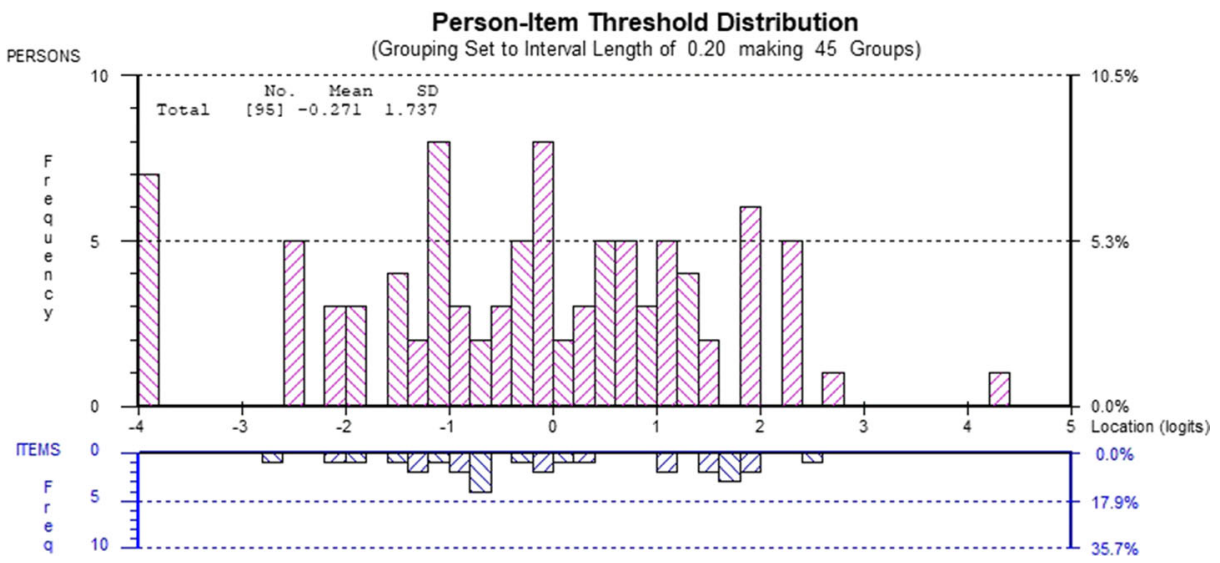

Fig. 1 Person-item distribution for the Tamil version of the FCV-19S 
well, with no item misfit or differential item functioning by demographic variables. Item means, skewness, and kurtosis were comparable to those of other international studies, particularly when compared with the Bangla version or a sample from India that had completed the English-language version. Internal consistency reliability of the Tamil version of the FCV-19S was also excellent, even exceeding the values obtained in other language versions, making the tool both valid and reliable for the assessment of fear of COVID-19 suitable for both between-group and within-participant comparisons in Tamil-speaking populations. Due to some floor effects, the FCV-19S may fail to differentiate between individuals who have very low levels of fear.

Our study replicated common reports that the FCV-19S provides a unidimensional assessment of fear of COVID-19 (Ahorsu et al., 2020; Alyami et al., 2020; Perz et al., 2020; Sakib et al., 2020; Satici et al., 2020; Soraci et al., 2020; Tsipropoulou et al., 2020). The only exception is a study reporting on results from a Hebrew version (Bitan et al., 2020) where the authors proposed that items 1, 2, 4, and 5 (generally afraid, uncomfortable thinking about COVID-19, afraid of losing life, anxious from news) form a factor called emotional fear reactions and that items 3 , 6 , and 7 (clammy hands, lack of sleep, heart racing) be grouped into a factor called symptomatic expressions of fear. Pakpour et al. (2020) argued that the statistical approach used by Bitan et al. (2020) was inappropriate and that a confirmatory factor analysis should have been used instead of a principal components analysis where a two-factor was forced even though a single-factor solution had already indicated that a fit was adequate. In their validation of the Arabic version of the FCV-19S, Alyami et al. (2020) noted error co-variance for items 3, 6, and 7, although this could be resolved by correlating the error co-variance in a confirmatory factor analysis. The present study of the Tamil version also noted some residual correlations that indicate a clustering of items 3, 6, and 7. However, these relationships were not substantial enough to challenge the unidimensionality of the scale. As shown in our overview of various studies (Table 2), these three items tend to exhibit floor effects. This was particularly the case in the study by Bitan et al. (2020), which indicates that the clustering of items 3, 6, and 7 may be due to sample-related method effects. This was not the case for the present Tamil version, which may be used with confidence as a unidimensional scale.

The following limitations of the present study need to be noted. Firstly, although the present sample size met the minimum requirements for Rasch analysis, collection of more data using this scale would provide more accurate reference mean scores for future comparative work using the Tamil version. Such work may also attempt to achieve better representation by demographic factors than achieved by the snowball sampling of the present study. Secondly, the present psychometric analysis was limited to residents in India, and further studies may also explore the suitability of the scale in other countries with significant Tamil-speaking populations, including Sri Lanka, Malaysia, and Singapore. Lastly, in order to reduce response burden for participants, the present study did not collect measures of stress and anxiety as a test of construct validity. However, this was deemed redundant given that the correlations between relevant constructs and the FCV-19S have been demonstrated repeatedly and consistently for a number of language versions (Ahorsu et al., 2020; Alyami et al., 2020; Huarcaya-Victoria et al., 2020; Perz et al., 2020; Satici et al., 2020; Soraci et al., 2020; Tsipropoulou et al., 2020). 


\section{Appendix. Tamil Version of the Fear of COVID-19 Scale}

\section{கொரோனா வைரஸ்-19 பற்றிய உளவியல் நிலைப்பாடு அறியும்}

\section{ஆராய்ச்சி படிவம்}

1. கொரோனா வைரஸ்-19 க்கு நான் மிகவும் பயப்படுகிறேன்

$\square$ சிறிதும் ஒப்புக்கொள்ளவில்லை

$\square$ ஓப்புக்கொள்ளவில்லை

$\square$ தெரியவில்லை

$\square$ ஒப்புக்கொள்கிறேன்

$\square$ முற்றிலும் ஒப்புக்கொள்கிறேன்

2. கொரோனா வைரஸ்-19 தொற்று பற்றி சந்திக்க எனக்கு சங்கடமாக இருக்கிறது

சிறிதும் ஓப்புக்கொள்ளவில்லை

$\square$ ஓப்புக்கொள்ளவில்லை

$\square$ தெரியவில்லை

$\square$ ஒப்புக்கொள்கிறேன்

$\square$ முற்றிலும் ஒப்புக்கொள்கிறேன்

3. கொரோனா வைரஸ்-19 பற்றி நினைக்கும் போது என் கைகள் வியர்வையால் ஈரமாகின்றன

$\square$ சிறிதும் ஓப்புக்கொள்ளவில்லை

$\square$ ஒப்புக்கொள்ளவில்லை

$\square$ தெரியவில்லை

ஓப்புக்கொள்கிறேன்

$\square$ முற்றிலும் ஒப்புக்கொள்கிறேன்

4. கொரோனா வைரஸ்-19 தொற்று காரணமாக என் உயிரை இழக்க நேரிடுமோ என்று நான் பயப்படுகிறேன் 
சிறிதும் ஒப்புக்கொள்ளவில்லை

$\square$ ஒப்புக்கொள்ளவில்லை

$\square$ தெரியவில்லை

$\square$ ஒப்புக்கொள்கிறேன்

$\square$ முற்றிலும் ஒப்புக்கொள்கிறேன்

5. சமூக ஊடகங்களில் கொரோனா வைரஸ்-19 பற்றிய செய்திகளையும், நிகழ்வுகளையும் பார்க்கும்போது நான் பதற்றமடைகிறேன் அல்லது பயப்படுகிறேன்

$\square$ சிறிதும் ஒப்புக்கொள்ளவில்லை

$\square$ ஒப்புக்கொள்ளவில்லை

தெரியவில்லை

$\square$ ஒப்புக்கொள்கிறேன்

$\square$ முற்றிலும் ஒப்புக்கொள்கிறேன்

6. கொரோனா வைரஸ்-19 தொற்று ஏற்பட்டுவிடுமோ என்ற பயத்தால் என் தூக்கம் பாதிக்கப்படுகிறது

சிறிதும் ஒப்புக்கொள்ளவில்லை

ஒப்புக்கொள்ளவில்லை

தெரியவில்லை

$\square$ ஒப்புக்கொள்கறேன்

$\square$ முற்றிலும் ஒப்புக்கொள்கிறேன்

7. கொரோனா வைரஸ்-19 தொற்று ஏற்பட்டுவிடுமோ என்று நினைக்கும் போது என் இதயம் வேகமாக துடிக்கிறது

சிறிதும் ஒப்புக்கொள்ளவில்லை

ஓப்புக்கொள்ளவில்லை

தெரியவில்லை

ஒ ஒப்புக்கொள்கிறேன்

$\square$ முற்றிலும் ஒப்புக்கொள்கிறேன் 
Acknowledgements We thank the participants and Sundeep Ananda, Chairman of Bharath Institute for Higher Learning and Research, for his support.

\section{Declarations}

Ethics Approval All procedures followed were in accordance with the ethical standards of the responsible committee on human experimentation (institutional and national) and with the Helsinki Declaration.

Informed Consent All participants provided electronic informed consent.

Conflict of Interest The authors declare no competing interests.

\section{References}

Ahorsu, D. K., Lin, C.-Y., Imani, V., Saffari, M., Griffiths, M. D., \& Pakpour, A. H. (2020). The fear of COVID19 scale: Development and initial validation. International Journal of Mental Health and Addiction. https:// doi.org/10.1007/s11469-020-00270-8.

Alyami, M., Henning, M., Krägeloh, C. U., \& Alyami, H. (2020). Psychometric evaluation of the Arabic version of the fear of COVID-19 scale. International Journal of Mental Health and Addiction. https://doi.org/10. 1007/s11469-020-00316-X.

Andrich, D., Sheridan, B., \& Luo, G. (2009). RUMM 2030. RUMM Laboratory.

Beaton, D. E., Bombardier, C., Guillemin, F., \& Ferraz, M. B. (2000). Guidelines for the process of cross-cultural adaptation of self-report measures. Spine, 25(24), 3186-3191. https://doi.org/10.1097/00007632200012150-00014.

Bitan, D. T., Grossman-Giron, A., Bloch, Y., Mayer, Y., Shiffman, N., \& Mendlovic, S. (2020). Fear of COVID19 scale: Psychometric characteristics, reliability and validity in the Israeli population. Psychiatry Research, 289, 113100. https://doi.org/10.1016/j.psychres.2020.113100.

Britannica.com. (2021). Tamil language. https://www.britannica.com/topic/Tamil-language (last accessed 26 February 2021)

Bukhari, E. E., Temsah, M. H., Aleyadhy, A. A., Alrabiaa, A. A., Alhboob, A. A., Jamal, A. A., \& Binsaeed, A. A. (2016). Middle East respiratory syndrome coronavirus (MERS-CoV) outbreak perceptions of risk and stress evaluation in nurses. Journal of Infection in Developing Countries, 10(8), 845-850. https://doi.org/10. 3855/jidc.6925.

Byrne, B. M., \& Campbell, T. L. (1999). Cross-cultural comparisons and the presumption of equivalent measurement and theoretical structure: A look beneath the surface. Journal of Cross-Cultural Psychology, 30(5), 555-574. https://doi.org/10.1177/0022022199030005001.

National Informatics Centre. (2020). Government of Tamil Nadu. https://www.tn.gov.in/tamilnadustate (last accessed 26 Feb 2021)

Department of Statistics Singapore. (2021). Literacy and language. https://www.singstat.gov.sg/-/media/files/ publications/cop2000/census 2000 advance data release/chap4.pdf (last accessed 26 Feb 2021)

DeVellis, R. F. (2016). Scale development: Theory and applications. SAGE.

Doshi, D., Karunakar, P., Sukhabogi, J. R., Prasanna, J. S., \& Mahajan, S. V. (2020). Assessing coronavirus fear in Indian population using the fear of COVID-19 scale. International Journal of Mental Health and Addiction. https://doi.org/10.1007/s11469-020-00332-x.

Encyclopedia.com. (2021). Tamil of Sri Lanka. https://www.encyclopedia.com/places/asia/sri-lankan-politicalgeography/tamil-sri-lanka (last accessed 26 Feb 2021)

Ferketich, S. (1991). Focus on psychometrics. Aspects of item analysis. Research in Nursing \& Health, 14(2), 165-168. https://doi.org/10.1002/nur.4770140211.

Galea, S., Merchant, R. M., \& Lurie, N. (2020). The mental health consequences of COVID-19 and physical distancing: The need for prevention and early intervention. JAMA Internal Medicine, 180, 817-818. https:// doi.org/10.1001/jamainternmed.2020.1562.

Gritsenko, V., Skugarevsky, O., Konstantinov, V., Khamenka, N., Marinova, T., Reznik, A., \& Isralowitz, R. (2020). COVID 19 fear, stress, anxiety, and substance use among Russian and Belarusian university students. International Journal of Mental Health and Addiction. https://doi.org/10.1007/s11469-02000330-z. 
Harper, C. A., Satchell, L. P., Fido, D., \& Latzman, R. D. (2020). Functional fear predicts public health compliance in the COVID-19 pandemic. International Journal of Mental Health and Addiction. https:// doi.org/10.1007/s11469-020-00281-5.

Huarcaya-Victoria, J., Villarreal-Zegarra, D., Podestà, A., \& Luna-Cuadros, M. A. (2020). Psychometric properties of a Spanish version of the fear of COVID-19 scale in general population of Lima Peru. International Journal of Mental Health and Addiction. https://doi.org/10.1007/s11469-020-00354-5.

Johns Hopkins University \& Medicine. (2020). Johns Hopkins Coronavirus Resource Center. https:// coronavirus.jhu.edu/map.html

Knipe, D., Evans, H., Marchant, A., Gunnell, D., \& John, A. (2020). Mapping population mental health concerns related to COVID-19 and the consequences of physical distancing: A Google trends analysis. Wellcome Open Research, 5, 82-92. https://doi.org/10.12688/wellcomeopenres.15870.1.

Krägeloh, C. U., Medvedev, O. N., Hill, E. M., Webster, C. S., Booth, R. J., \& Henning, M. A. (2019). Improving measurement of trait competitiveness: a Rasch analysis of the Revised Competitiveness Index with samples from New Zealand and US university students. Psychological Reports, 122(2), 689-708. https://doi.org/10.1177/0033294118762548.

Linacre, J. M. (1994). Sample size and item calibration stability. Rasch Measurement Transactions, 7(4), 328.

Lu, W., Wang, H., Lin, Y., \& Li, L. (2020). Psychological status of medical workforce during the COVID-19 pandemic: A cross-sectional study. Psychiatry Research, 288, 112936. https://doi.org/10.1016/j.psychres. 2020.112936.

Mahmood, Q. K., Jafree, S. R., \& Qureshi, W. A. (2020). The psychometric validation of FCV19S in Urdu and sociodemographic association with fear in the people of the Khyber Pakhtunkhwa (KPK) province in Pakistan. International Journal of Mental Health and Addiction. https://doi.org/10.1007/s11469-020-00371-4.

Masuyama, A., Shinkawa, H., \& Kubo, T. (2020). Validation and psychometric properties of the Japanese version of the fear of COVID-19 scale among adolescents. International Journal of Mental Health and Addiction. https://doi.org/10.1007/s11469-020-00368-z.

Pakpour, A. H., Griffiths, M. D., \& Lin, C.-Y. (2020). Assessing the psychological response to the COVID-19: A response to Bitan et al. "Fear of COVID-19 scale: Psychometric characteristics, reliability and validity in the Israeli population". Psychiatry Research, 290, 113127. https://doi.org/10.1016/j.psychres.2020.113127.

Pang, N. T. P., Kamu, A., Hambali, N. L. B., Mun, H. C., Kassim, M. A., Mohamed, N. H., Ayu, F., Rahim, S. S. S. A., Omar, A., \& Jefree, M. S. (2020). Malay version of the fear of COVID-19 scale: Validity and reliability. International Journal of Mental Health and Addiction. https://doi.org/10.1007/s11469-020$00355-4$.

Perz, C. A., Lang, B. A., \& Harrington, R. (2020). Validation of the fear of COVID-19 scale in a US college sample. International Journal of Mental Health and Addiction. https://doi.org/10.1007/s11469-020-00356-3.

Rajkumar, R. P. (2020). COVID-19 and mental health: A review of the existing literature. Asian Journal of Psychiatry, 52, 102066. https://doi.org/10.1016/j.ajp.2020.102066.

Reynolds, D. L., Garay, J. R., Deamond, S. L., Moran, M. K., Gold, W., \& Styra, R. (2008). Understanding, compliance and psychological impact of the SARS quarantine experience. Epidemiology and Infection, 136(7), 997-1007. https://doi.org/10.1017/S0950268807009156.

Reznik, A., Gritsenko, V., Konstantinov, V., Khamenka, N., \& Isralowitz, R. (2020). COVID-19 fear in Eastern Europe: Validation of the fear of COVID-19 scale. International Journal of Mental Health and Addiction. https://doi.org/10.1007/s11469-020-00283-3.

Sakib, N., Mamun, M. A., Bhuiyan, A. I., Hossain, S., Al Mamun, F., Hosen, I., Abdullah, A. H., Sarker, A., Mohiuddin, M. S., Rayhan, I., Hossain, M., Sikder, T., Gozal, D., Muhit, M. A., Islam, S. M. S., Griffiths, M. D., \& Pakpour, A. H. (2020). Psychometric validation of the Bangla fear of COVID-19 scale: Confirmatory factor analysis and Rasch analysis. International Journal of Mental Health and Addiction. https://doi.org/10.1007/s11469-020-00289-x.

Satici, B., Gocet-Tekin, E., Deniz, M. E., \& Satici, S. A. (2020). Adaptation of the fear of COVID-19 scale: Its association with psychological distress and life satisfaction in Turkey. International Journal of Mental Health and Addiction. https://doi.org/10.1007/s11469-020-00294-0.

Smith, E. V. (2002). Detecting and evaluation the impact of multidimensionality using item fit statistics and principal component analysis of residuals. Journal of Applied Measurement, 3(2), 205-231.

Soraci, P., Ferrari, A., Abbiati, F. A., Del Fante, E., De Pace, R., Urso, A., \& Griffiths, M. D. (2020). Validation and psychometric evaluation of the Italian version of the fear of COVID-19 scale. International Journal of Mental Health and Addiction. https://doi.org/10.1007/s11469-020-00277-1.

Tennant, A., \& Conaghan, P. G. (2007). The Rasch measurement model in rheumatology: What is it and why use it? When should it be applied, and what should one look for in a Rasch paper? Arthritis and Rheumatism, 57(8), 1358-1362. https://doi.org/10.1002/art.23108. 
Tsipropoulou, V., Nikopoulou, V. A., Holeva, V., Nasika, Z., Diakogiannis, I., Sakka, S., Kostikidou, S., Varvara, C., Spyridopoulou, E., \& Parlapani, E. (2020). Psychometric properties of the Greek version of FCV-19S. International Journal of Mental Health and Addiction. https://doi.org/10.1007/s11469-020-00319-8.

Willse, J. T. (2017). Polytomous Rasch models in counseling assessment. Measurement and Evaluation in Counseling and Development, 50(4), 248-255. https://doi.org/10.1080/07481756.2017.1362656.

Winter, T., Riordan, B. C., Pakpour, A. H., Griffiths, M. D., Mason, A., Poulgrain, J. W., \& Scarf, D. (2020). Evaluation of the English version of the fear of COVID-19 scale and its relationship with behavior change and political beliefs. International Journal of Mental Health and Addiction. https://doi.org/10.1007/s11469020-00342-9.

Worldometer. (2020). Countries where Coronavirus has spread. https://www.worldometers.info/coronavirus/ countries-where-coronavirus-has-spread/

Zolotov, Y., Reznik, A., Bender, S., \& Isralowitz, R. (2020). COVID-19 fear, mental health, and substance use among Israeli university students. International Journal of Mental Health and Addiction. https://doi.org/10. 1007/s11469-020-00351-8.

Publisher's Note Springer Nature remains neutral with regard to jurisdictional claims in published maps and institutional affiliations. 